\title{
Radial velocity survey for planets and brown dwarf companions to very young brown dwarfs and very low-mass stars in Chamaeleon I with UVES at the VLT ${ }^{\star, \star \star}$
}

\author{
V. Joergens
}

Leiden Observatory / Sterrewacht Leiden, PO Box 9513, 2300 RA Leiden, Netherlands

e-mail: viki@strw.leidenuniv.nl

Received 11 May 2005 / Accepted 6 September 2005

ABSTRACT

We present results of a radial velocity $(R V)$ survey for planets and brown dwarf (BD) companions to very young BDs and (very) low-mass stars in the Cha I star-forming cloud. Time-resolved high-resolution echelle spectra of Cha H $\alpha$ 1-8 and Cha H $\alpha$ 12 (M6-M8), B34 (M5), CHXR 74 (M4.5), and Sz 23 (M2.5) were taken with UVES at the VLT between 2000 and 2004. The precision achieved for the relative $R V \mathrm{~s}$ range between 40 and $670 \mathrm{~m} \mathrm{~s}^{-1}$ and is sufficient to detect Jupiter mass planets around the targets. This is the first $R V$ survey of very young BDs. It probes multiplicity, which is a key parameter for formation in an as yet unexplored domain, in terms of age, mass, and orbital separation. We find that the subsample of ten BDs and very low-mass stars (VLMSs, $M \lessgtr 0.12 M_{\odot}$, spectral types M5-M8) has constant $R V \mathrm{~s}$ on time scales of 40 days and less. For this group, estimates of upper limits for masses of hypothetical companions range between $0.1 M_{\text {Jup }}$ and $1.5 M_{\text {Jup }}$ for assumed orbital separations of $0.1 \mathrm{AU}$. This hints at a rather small multiplicity fraction for very young BDs/VLMSs, for orbital separations of $\lesssim 0.1 \mathrm{AU}$ and orbital periods of $\lesssim 40$ days. Furthermore, the non-variable objects demonstrate the lack of any significant $R V$ noise due to stellar activity down to the precision necessary to detect giant planets. Thus, very young BDs/VLMSs are suitable targets for $R V$ surveys for planets. Three objects of the sample exhibit significant $R V$ variations with peak-to-peak $R V$ differences of $2-3 \mathrm{~km} \mathrm{~s}^{-1}$. For the highest mass object observed with UVES $\left(\mathrm{Sz} 23, \sim 0.3 M_{\odot}\right.$ ), the variations are on time scales of days, which might be explained by rotational modulation. On the other hand, the BD candidate $\mathrm{Cha} \mathrm{H} \alpha$ (M6.5) and the low-mass star CHXR 74 (M4.5) both display significant $R V$ variations on times scales of $\gtrsim 150$ days, while they are both $R V$ constant or show only much smaller amplitude variations on time scales of days to weeks, i.e. of the rotation periods. A suggested explanation for the detected $R V$ variations of CHXR 74 and $\mathrm{Cha} \mathrm{H} \alpha 8$ is that they are caused by giant planets or very low-mass BDs of at least a few Jupiter masses orbiting with periods of several months or longer. Thus, the presented $R V$ data indicate that orbital periods of companions to very young BDs and (very) low-mass stars are possibly several months or longer, and that orbital separations are $\gtrsim 0.2 \mathrm{AU}$. This parameter range has not been covered for all targets yet, but will be probed by follow-up observations. Furthermore, we show that the scaled down equivalent to the BD desert found around solar-like stars would be a giant planet desert around BD and VLMS primaries, if formed by the same mechanism. The present data test its existence for the targets in the limited separation range of the survey. So far, no hints of companions in a "giant planet desert" have been found.

Key words. stars: low-mass, brown dwarfs - stars: pre-main sequence - binaries: spectroscopic - techniques: radial velocities planetary systems

\section{Introduction}

In the last ten years, more than 150 extrasolar planets have been detected by radial velocity $(R V)$ surveys (e.g. Moutou et al. 2005; Marcy et al. 2005a for recent discoveries). The $R V$ technique traces periodic $R V$ variabilities caused by the wobble of the primary object induced by an orbiting mass. Other sources of $R V$ variability, like surface activity, can mimick a

\footnotetext{
* Based on observations obtained at the Very Large Telescope of the European Southern Observatory at Paranal, Chile in program 65.L-0629, 65.I-0011, 268.D-5746, 72.C-0653.

$\star \star$ Appendix A is only available in electronic form at http://www.edpsciences.org
}

companion. Therefore, up to now $R V$ surveys for planets have been restricted to considerably old primaries with ages on the order of a few billion years and with mainly solar-like spectral types. The youngest $R V$ planet known to date is orbiting the zero-age main sequence star $\iota$ Hor with an estimated age in the range of $30 \mathrm{Myr}$ to $2 \mathrm{Gyr}$ (Kürster et al. 2000). Very recently, evidence of substellar companions, possibly of planetary mass, around the young BD 2M1207 A (age 5-12 Myr, Chauvin et al. 2004, 2005) and around the very young star GQ Lup (age 0.1-2 Myr, Neuhäuser et al. 2005) has been found from direct AO imaging. Furthermore, most planets known to date orbit around solar-mass stars with spectral types of late-F, $\mathrm{G}$, and early-K with the exception of two planets around the 
M4-dwarf G1 876 (Delfosse et al. 1998; Marcy et al. 1998, 2001), a planet around the M2.5 dwarf GJ 436 (Santos et al. 2004; Butler et al. 2004) and the 2M1207 system (see above).

Further progress in the field of extrasolar planets is expected from the search for planets around very young, as well as very low-mass primaries. The detection of young planets and a census of planets around stars of all spectral types and even around BDs, is an important step towards understanding planet formation.

Detection of planets around BDs, as well as of young $\mathrm{BD}$ binaries (BD-BD pairs), would, on the other hand, constrain the formation of BDs (see Joergens 2005, for a recent review of $\mathrm{BD}$ formation). Besides indications of a possible planetary mass object in orbit around 2M1207 (Chauvin et al. 2005), no planet of a BD has been found yet. In recent years, several BD binaries have been detected in the field, mainly by direct imaging (e.g. Martín et al. 1999, 2000; Koerner et al. 1999; Reid et al. 2001; Lane et al. 2001; Kenworthy et al. 2001; Close et al. 2002a,b; Bouy et al. 2003) and about three by spectroscopic surveys (Basri \& Martín 1999; Guenther \& Wuchterl 2003). These detections of companions to nearby field BDs give important insight into the substellar binary population at ages of a few billion years. However, these results represent only a boundary condition, which is not necessarily matched at any earlier time. Therefore, it is useful for the current discussion of BD formation scenarios to study multiplicity at very young ages. However, besides indications from direct imaging for the binarity of Cha $\mathrm{H} \alpha 2$ (Neuhäuser et al. 2002) and 2M 1101-7732 (Luhman 2004) in the Cha I cloud and of DENIS-P J18590.9-370632 in the R-CrA star-forming region (Bouy et al. 2004), all other known BD binaries are fairly old.

In order to probe both BD multiplicity at a very young age and the occurrence of planets around very young and very low-mass (substellar) primaries, we initiated an $R V$ survey in the Cha I star-forming cloud with the UV-Visual Echelle Spectrograph (UVES) at the Very Large Telescope (VLT). The targets are very young BDs and (very) low-mass stars in the center of Cha I at an age of only a few million years. This is the first systematic $R V$ survey for companions around young BDs and VLMSs. We present evidence in this paper that they show only very small amplitude $R V$ variability due to activity and that they are suitable targets for $R V$ planet surveys. This opens up a new parameter range for the search for extrasolar planets, namely looking for very young planets at very close separations. Thus, the initiated $R V$ survey studies the existence of companions in what is an as yet unexplored domain, not only in terms of primary masses (substellar regime) and ages (a few million years), but also in terms of companion masses (sensitive down to planetary masses) and separations (smaller than about $2 \mathrm{AU}$ ). It might sample a substantially different companion formation mechanism than the one represented by BD binaries detected so far by direct imaging.

First results were obtained by Joergens \& Guenther (2001) within the framework of this survey based on UVES spectra taken in 2000 on mean $R V \mathrm{~s}$, projected rotational velocities $v \sin i$, and lithium absorption, as well as on the kinematics of the BD population in Cha I in comparison with that of $\mathrm{T}$ Tauri stars in the same region. The data analysis was improved, and revised $R V \mathrm{~s}$ were then measured by Joergens (2003). Additional UVES spectra were taken in 2002 and 2004. In the paper on hand, the time-resolved $R V \mathrm{~s}$ measured from all UVES spectra taken during this survey between 2000 and 2004 are finally presented and analysed in terms of a search for spectroscopic companions down to planetary masses. This enlargement of the data set and the improved data reduction allowed an improved kinematic study of very young BDs based on the mean $R V$ s measured with UVES, published elsewhere (Joergens 2006).

The paper is organized as follows: Sect. 2 introduces the observed sample of BDs and (very) low-mass stars in Cha I. In Sects. 3 and 4, the acquisition and reduction of high-resolution UVES spectra and the measurement of $R V \mathrm{~s}$ are described. In Sect. 5, the results are presented and discussed. Finally, Sect. 6 contains conclusions and a summary.

\section{Sample}

The targets of this $R V$ survey are BDs and (very) low-mass stars with an age of a few million years situated in the center of the nearby $(\sim 160 \mathrm{pc})$ Cha I star-forming cloud (Comerón et al. 1999, 2000; Neuhäuser \& Comerón 1998, 1999). Membership in the Cha I cluster and, therefore, the youth of the objects, is well established based on $\mathrm{H} \alpha$ emission, lithium absorption, spectral types, and $R V \mathrm{~s}$ (see references above, Joergens \& Guenther 2001; Joergens 2006).

UVES spectroscopy has been performed so far for Cha $\mathrm{H} \alpha$ 1-8 and Cha $\mathrm{H} \alpha 12$, B34, CHXR 74, and Sz 23. Two of them (Cha $\mathrm{H} \alpha 1,7)$ are classified as bona fide BDs (M7.5-M8) with mass estimates of $30-40 M_{\odot}$, five (Cha H $\alpha 2,3,6$, $8,12)$ as BD candidates (M6.5-M7) with mass estimates of 50-70 $M_{\odot}$, two (Cha $\left.\mathrm{H} \alpha 4,5\right)$ as VLMSs (M6) with masses close to the substellar borderline $\left(\sim 0.1 M_{\odot}\right)$, one (B 34) as VLMS (M5) with a mass estimate of $0.12 M_{\odot}$, and two (CHXR 74, Sz 23) as low-mass T Tauri stars (M4.5, M2.5) with $0.17 M_{\odot}$ and $0.3 M_{\odot}$, resp.

\section{Acquisition and reduction of UVES spectra}

High-resolution spectra have been taken so far for twelve BDs and (very) low-mass stars in Cha I between the years 2000 and 2004 with the cross-dispersed echelle spectrograph UVES (Dekker et al. 2000) attached to the $8.2 \mathrm{~m}$ Kueyen telescope of the VLT operated by the European Southern Observatory at Paranal, Chile. For each object, at least two spectra separated by a few weeks have been obtained in order to monitor time dependence of the $R V \mathrm{~s}$. For several objects, more than two and up to twelve spectra were taken.

The observations were performed with the red arm of the two-armed UVES spectrograph equipped with a mosaic of two CCDs. The mosaic is made of a $2 \mathrm{~K} \times 4 \mathrm{~K}$ EEV chip (pixel size $15 \mu \mathrm{m}$ ) for the blue part of the red arm and an MIT-LL $\mathrm{CCD}$ for the red part of the red arm. The wavelength regime from $6600 \AA$ to $10400 \AA$ was covered with a spectral resolution of $\lambda / \Delta \lambda=40000$. A slit of $1^{\prime \prime}$ to $1.2^{\prime \prime}$ was used.

A standard CCD reduction of the two-dimensional UVES echelle frames, including bias correction, flat fielding and 
cosmic ray elimination, was performed with $\mathrm{IRAF}^{1}$. The flat field correction of the small-scale pixel-to-pixel variations of the CCD was performed with a master flat field frame created by taking the median of several flat field exposures. The master flat was normalized, in order to remove large-scale structures by fitting its intensity along the dispersion by a third order fit, while setting all points outside the order aperture to 1 and dividing the master flat by this fit.

Subsequently, one-dimensional spectra were extracted, including a correction for sky background light. Compared to the previous reduction of UVES spectra by Joergens \& Guenther (2001), this reduction step was improved, as in Guenther \& Wuchterl (2003), by extracting first each echelle order as a twodimensional spectrum, then performing the sky subtraction on these $2 \mathrm{D}$ frames, and finally extracting the one-dimensional science spectrum. No rebinning was done in order to achieve a high $R V$ precision.

Finally, the spectra were wavelength calibrated using the echelle package of IRAF. This was done in a first step by the use of Thorium-Argon arc spectra. In order to achieve a high wavelength and therefore $R V$ precision, an additional correction by means of telluric $\mathrm{O}_{2}$ lines ( $B$-band centered at $6880 \AA$ ) produced in the Earth's atmosphere was applied. It has been shown that they are stable up to $\sim 20 \mathrm{~m} \mathrm{~s}^{-1}$ (Balthasar et al. 1982; Caccin et al. 1985).

\section{Radial velocities}

$R V \mathrm{~s}$ were determined by a cross-correlation of plenty of stellar lines of the object spectra against a template spectrum and locating the correlation maximum. For measuring Doppler shifts of stellar features, appropriate wavelength regions were selected that are not affected by telluric lines, cosmetic defects of the CCD, or fringes of the CCD in the near-IR. A heliocentric correction was applied to the observed $R V \mathrm{~s}$. In several cases, the $R V$ derived for one night was based on two consecutive single spectra to provide two independent measurements of the $R V$. This allows a solid estimation of the error of the relative $R V \mathrm{~s}$ based on the standard deviation for two such data points. Tables 1 and 2 list the resulting heliocentric $R V \mathrm{~s}$, error estimates, and a mean $R V$ for each target. $R V$ values based on two consecutive single spectra obtained in the same night are marked with an asterisk in the last column of these tables. These error measurements depend linearly, as expected, on the signal-to-noise $(\mathrm{S} / \mathrm{N})$ of the spectra. This linear relationship is used in turn to estimate errors for $R V$ data points, which are based on only one measurement per night. An $R V$ precision between $40 \mathrm{~m} \mathrm{~s}^{-1}$ and $670 \mathrm{~m} \mathrm{~s}^{-1}$, depending on the $\mathrm{S} / \mathrm{N}$ of the individual spectra, was achieved (last column of Tables 1 and 2). We note that the precision of the $R V \mathrm{~s}$ is limited by the $\mathrm{S} / \mathrm{N}$ of the spectra and not by systematic effects. The relatively high precision that was achieved for the relative velocities does not apply to the absolute velocities due to additional uncertainties in the zero point. A mean UVES spectrum of selected high

${ }^{1}$ IRAF is distributed by the National Optical Astronomy Observatories, which is operated by the Association of Universities for Research in Astronomy, Inc. (AURA) under cooperative agreement with the National Science Foundation.
S/N spectra of the very low-mass M6-type star Cha $\mathrm{H} \alpha 4$ served as a template. The zero point of the velocity was determined based on a fit to the blend of the prominent lithium lines at $\lambda \lambda 6707.76$ and $6707.93 \AA$ in three different high $\mathrm{S} / \mathrm{N}$ spectra. The standard deviation of these fits of $400 \mathrm{~m} \mathrm{~s}^{-1}$ was assumed as an additional error for the absolute velocities. An observing $\log$ listing all individual measured $R V \mathrm{~s}$ is given in Tables A.1 and A.2 in the Appendix.

\section{Results}

The monitored $R V \mathrm{~s}$ are constant within the measurement errors for the majority of the observed BDs and (very) low-mass stars, whereas for three of the targets, our observations reveal significant $R V$ variability. The measured $R V \mathrm{~s}$ are listed in Tables 1 and 2 and plotted in Figs. 3-9. They are presented in detail in Sects. 5.2 and 5.3 after elaborating on the probed companion masses and orbital separations in the following section.

\subsection{Covered parameter space}

For Cha $\mathrm{H} \alpha 1,2,3,5,6,7,8$, and 12, two $R V$ measurements were obtained with a $\sim 20$ day time offset, while for $\mathrm{Cha} \mathrm{H} \alpha 4$, Cha $\mathrm{H} \alpha$ 8, B 34, CHXR 74, and Sz 23, four to five $R V$ points were obtained with sampling intervals between 5 and 70 days. In addition, follow-up observations were also performed for four targets: for Cha $\mathrm{H} \alpha 4$, Cha $\mathrm{H} \alpha 8$ after two years and for CHXR 74, Sz 23 after four years.

Figures 1 and 2 illustrate the probed parameter space in terms of companion masses, orbital separations, and periods for primaries of $0.1 M_{\odot}$ and $0.06 M_{\odot}$, respectively. For targets with a mass of about $0.1 M_{\odot}(\mathrm{ChaH} \alpha 4,5$, B 34), an average $R V$ precision of $190 \mathrm{~m} \mathrm{~s}^{-1}$ was achieved in this survey. It can be seen from Fig. 1 (upper panel and middle sketch) that this basically allows a $1 M_{\text {Jup }}$ companion to be detected at separations of $0.25 \mathrm{AU}$ or smaller, while a $3 M_{\text {Jup }}$ companion can be detected out to $2 \mathrm{AU}$, and companions with $\geq 5 M_{\text {Jup }}$ at least out to $4 \mathrm{AU}^{2}$. However, due to a limited time base, the whole possible separation range has not been covered yet. The orbital periods sampled in this survey so far vary among the different targets and are between 40 days (a time interval of 20 days allows detection of an orbital period twice as long) and 8 years. As displayed in the lower panel of Fig. 1, this period sampling allows substellar companions to be detected at separations between $0.1 \mathrm{AU}$ and about $2 \mathrm{AU}$ relatively independent of companion mass.

Figure 2 shows the situation for a lower mass primary, here for $0.06 M_{\odot}$. With the average $R V$ precision of $390 \mathrm{~m} \mathrm{~s}^{-1}$ achieved for primaries of about $(0.06 \pm 0.01) M_{\odot}(\mathrm{ChaH} \alpha 2$, $3,6,8,12)$, a $1 M_{\text {Jup }}$ companion is basically detectable out to $0.1 \mathrm{AU}$, a $3 M_{\text {Jup }}$ companion out to $0.8 \mathrm{AU}$ and companions with $\geq 6 M_{\text {Jup }}$ at least out to $3 \mathrm{AU}$. Based on the sampled orbital periods between 40 days and 8 years, companions could have been detected so far out to $0.1 \mathrm{AU}$ for all targets in this

\footnotetext{
2 It is noted that the average $R V$ precision for B 34 alone is significantly better $\left(90 \mathrm{~m} \mathrm{~s}^{-1}\right)$ and, therefore, allows detection of less massive companions at larger separations, e.g. $1 M_{\text {Jup }}$ out to $1 \mathrm{AU}$.
} 
Table 1. $R V \mathrm{~s}$ for BDs and VLMSs (M6-M8) in Cha I. Given are the date of the observation, heliocentric Julian day (HJD) at the middle of the exposure, the measured $R V$ and the estimated error $\sigma_{R V}$ of the relative $R V \mathrm{~s}$. The asterisk marks $R V \mathrm{~s}$ based on the average of two single measurements for which the errors are standard deviations. The last column gives the weighted mean $R V$ for the individual objects and the error of this mean, which takes into account an uncertainty of $400 \mathrm{~m} \mathrm{~s}^{-1}$ for the zero point of the velocity.

\begin{tabular}{|c|c|c|c|c|c|}
\hline Object & Date & HJD & $\begin{array}{c}R V \\
{\left[\mathrm{~km} \mathrm{~s}^{-1}\right]}\end{array}$ & $\begin{array}{l}\sigma_{R V} \\
{\left[\mathrm{~km} \mathrm{~s}^{-1}\right]}\end{array}$ & $\begin{array}{l}\overline{R V} \\
{\left[\mathrm{~km} \mathrm{~s}^{-1}\right]}\end{array}$ \\
\hline \multirow[t]{2}{*}{ Cha H $\alpha 1$} & 2000 Apr. 04 & 2451638.56395 & 16.167 & 0.53 & \multirow[b]{2}{*}{$16.35 \pm 0.63$} \\
\hline & 2000 Apr. 24 & 2451658.57346 & 16.648 & 0.67 & \\
\hline \multirow[t]{2}{*}{$\mathrm{Cha} \mathrm{H} \alpha 2$} & 2000 Apr. 04 & 2451638.59431 & 16.015 & 0.50 & \multirow[b]{2}{*}{$16.13 \pm 0.53$} \\
\hline & 2000 Apr. 24 & 2451658.60407 & 16.282 & 0.56 & \\
\hline \multirow[t]{2}{*}{ Cha $\mathrm{H} \alpha 3$} & 2000 Apr. 04 & 2451639.49340 & 14.357 & 0.45 & \multirow[b]{2}{*}{$14.56 \pm 0.60$} \\
\hline & 2000 Apr. 24 & 2451658.61991 & 14.758 & 0.45 & \\
\hline \multirow[t]{11}{*}{ Cha $\mathrm{H} \alpha 4$} & 2000 Mar. 14 & 2451617.73646 & 14.909 & $0.38 *$ & \multirow{11}{*}{$14.82 \pm 0.40$} \\
\hline & 2000 Mar. 24 & 2451627.80388 & 14.866 & $0.19 *$ & \\
\hline & 2000 Mar. 31 & 2451635.51085 & 14.773 & 0.14 & \\
\hline & 2000 Apr. 23 & 2451658.52150 & 14.908 & $0.05 *$ & \\
\hline & 2000 May 22 & 2451687.50595 & 14.830 & $0.08 *$ & \\
\hline & 2002 Jan. 17 & 2452291.78912 & 14.949 & $0.48 *$ & \\
\hline & 2002 Jan. 18 & 2452292.82508 & 14.754 & $0.12 *$ & \\
\hline & 2002 Jan. 24 & 2452298.70540 & 14.635 & $0.08 *$ & \\
\hline & 2002 Feb. 02 & 2452307.71979 & 15.064 & $0.39 *$ & \\
\hline & 2002 Feb. 04 & 2452309.74222 & 14.821 & $0.001 *$ & \\
\hline & 2002 Feb. 13 & 2452318.71915 & 14.985 & $0.07 *$ & \\
\hline \multirow[t]{2}{*}{ Cha $\mathrm{H} \alpha 5$} & 2000 Apr. 05 & 2451639.51485 & 15.499 & 0.45 & \multirow[b]{2}{*}{$15.47 \pm 0.43$} \\
\hline & 2000 Apr. 24 & 2451658.63522 & 15.446 & 0.42 & \\
\hline \multirow[t]{2}{*}{ Cha H $\alpha 6$} & 2000 Apr. 05 & 2451639.58967 & 16.093 & 0.50 & \multirow[b]{2}{*}{$16.37 \pm 0.68$} \\
\hline & 2000 Apr. 24 & 2451658.65099 & 16.652 & 0.50 & \\
\hline \multirow[t]{2}{*}{ Cha $\mathrm{H} \alpha 7$} & 2000 Apr. 05 & 2451639.55225 & 16.513 & 0.56 & \multirow[b]{2}{*}{$17.09 \pm 0.98$} \\
\hline & 2000 Apr. 24 & 2451658.68756 & 17.664 & 0.56 & \\
\hline \multirow[t]{6}{*}{ Cha $\mathrm{H} \alpha 8$} & 2000 Apr. 05 & 2451639.61095 & 14.787 & 0.50 & \multirow{5}{*}{$14.86 \pm 0.47(2000)$} \\
\hline & 2000 Apr. 24 & 2451658.72597 & 14.935 & 0.50 & \\
\hline & 2002 Mar. 06 & 2452339.68965 & 16.920 & $0.07 *$ & \\
\hline & 2002 Mar. 22 & 2452355.65264 & 16.912 & $0.16 *$ & \\
\hline & 2002 Apr. 16 & 2452380.61646 & 17.551 & $0.23 *$ & \\
\hline & 2002 Apr. 19 & 2452383.57565 & 17.379 & $0.03 *$ & $17.30 \pm 0.50(2002)$ \\
\hline \multirow[t]{2}{*}{ Cha H $\alpha 12$} & 2000 Apr. 05 & 2451639.63487 & 15.021 & 0.50 & \multirow[b]{2}{*}{$14.50 \pm 0.96$} \\
\hline & 2000 Apr. 25 & 2451659.59469 & 13.905 & 0.53 & \\
\hline
\end{tabular}

mass range and out to about 1.6 $\mathrm{AU}$ for $\mathrm{Cha} \mathrm{H} \alpha 8$ (Fig. 2, lower panel). While the $R V$ signal caused by an orbiting companion of a certain mass is generally larger for a lower mass primary than for a higher mass one, the significantly lower average $R V$ precision for primaries of $\sim 0.06 M_{\odot}\left(390 \mathrm{~m} \mathrm{~s}^{-1}\right)$ compared to $0.1 M_{\odot}$ $\left(190 \mathrm{~m} \mathrm{~s}^{-1}\right)$ restricts the accessible separation range.

To summarize, for all targets, separations $\lesssim 0.1 \mathrm{AU}$ ( $\sim 40$ day period) have been probed. In addition, for $\mathrm{Cha} \mathrm{H} \alpha 4$ and B 34 separations $\lesssim 0.25 \mathrm{AU}$ ( $\sim 150$ days) have been studied, for Cha $\mathrm{H} \alpha 4$ and Cha $\mathrm{H} \alpha 8 \lesssim 1-1.2 \mathrm{AU}$ ( $4 \mathrm{yr}$ ) and for CHXR 74 and $\mathrm{Sz} 23 \lesssim 2.5 \mathrm{AU}(8 \mathrm{yr})$.

\subsection{RV constant objects}

The $R V \mathrm{~s}$ for the BDs/VLMSs Cha $\mathrm{H} \alpha 1,2,3,4,5,6,7,12$, and B 34 are constant within the measurement uncertainties of $2 \sigma$ for $\mathrm{Cha} \mathrm{H} \alpha 4$ and of $1 \sigma$ for all others (Figs. 3-6). The sampling intervals vary among these objects. For the majority, it is $\$ 20$ days corresponding to $\$ 0.1 \mathrm{AU}$, while it is $\$ 70$ days ( $\lessgtr 0.25 \mathrm{AU}$ ) for B 34 and $\lesssim$ two years $(\lessgtr 1.2 \mathrm{AU})$ for Cha $\mathrm{H} \alpha 4$. From the non-detections of variability, we estimated upper limits for the projected masses $M_{2} \sin i$ of hypothetical companions for each object. Table 3 lists $R V$ differences for all 
Table 2. $R V$ s for (very) low-mass stars (M2.5-M5) in Cha I. See Table 1 for details.

\begin{tabular}{|c|c|c|c|c|c|}
\hline Object & $\overline{\text { Date }}$ & HJD & $\begin{array}{c}R V \\
{\left[\mathrm{~km} \mathrm{~s}^{-1}\right]}\end{array}$ & $\begin{array}{l}\sigma_{R V} \\
{\left[\mathrm{~km} \mathrm{~s}^{-1}\right]}\end{array}$ & $\begin{array}{l}\overline{R V} \\
{\left[\mathrm{~km} \mathrm{~s}^{-1}\right]}\end{array}$ \\
\hline \multirow[t]{5}{*}{ B 34} & 2000 Mar. 13 & 2451616.83205 & 15.795 & $0.09 *$ & \multirow{5}{*}{$15.75 \pm 0.42$} \\
\hline & 2000 Mar. 25 & 2451628.61377 & 15.746 & 0.04 & \\
\hline & 2000 Mar. 31 & 2451634.55482 & 15.749 & $0.09 *$ & \\
\hline & 2000 Apr. 23 & 2451657.53470 & 15.667 & $0.11 *$ & \\
\hline & 2000 May 22 & 2451686.51384 & 15.814 & $0.13 *$ & \\
\hline \multirow[t]{10}{*}{ CHXR 74} & 2000 Mar. 13 & 2451616.78715 & 15.376 & $0.09 *$ & \multirow{10}{*}{$14.58 \pm 0.62(2000)$} \\
\hline & 2000 Mar. 31 & 2451634.52092 & 14.499 & $0.05 *$ & \\
\hline & 2000 Apr. 22 & 2451656.51247 & 14.854 & $0.27 *$ & \\
\hline & 2000 May 21 & 2451686.48261 & 14.276 & $0.06 *$ & \\
\hline & 2004 Mar. 03 & 2453067.82956 & 17.196 & $0.05 *$ & \\
\hline & 2004 Mar. 12 & 2453076.66488 & 17.184 & $0.09 *$ & \\
\hline & 2004 Mar. 21 & 2453085.77022 & 17.009 & $0.29 *$ & \\
\hline & 2004 Mar. 24 & 2453088.79822 & 16.912 & $0.22 *$ & \\
\hline & 2004 Mar. 31 & 2453095.78042 & 17.454 & $0.01 *$ & \\
\hline & 2004 Apr. 01 & 2453096.77600 & 17.200 & $0.03 *$ & \\
\hline \multirow[t]{12}{*}{$\mathrm{Sz} 23$} & 2000 Mar. 14 & 2451617.68093 & 14.652 & 0.04 & \multirow{12}{*}{$15.57 \pm 0.55$} \\
\hline & 2000 Mar. 25 & 2451628.66914 & 15.926 & 0.07 & \\
\hline & 2000 Mar. 31 & 2451634.59142 & 15.564 & $0.13 *$ & \\
\hline & 2000 Apr. 22 & 2451657.49636 & 14.740 & $0.23 *$ & \\
\hline & 2000 May 20 & 2451685.48812 & 15.233 & $0.08 *$ & \\
\hline & 2004 Mar. 02 & 2453066.80358 & 15.152 & $0.39 *$ & \\
\hline & 2004 Mar. 08 & 2453072.84053 & 16.647 & $0.05 *$ & \\
\hline & 2004 Mar. 12 & 2453076.69419 & 16.377 & $0.13 *$ & \\
\hline & 2004 Mar. 21 & 2453085.80059 & 14.472 & $0.17 *$ & \\
\hline & 2004 Mar. 24 & 2453088.77028 & 16.360 & $0.10 *$ & \\
\hline & 2004 Mar. 31 & 2453095.75207 & 16.432 & $0.02 *$ & \\
\hline & 2004 Apr. 01 & 2453096.74897 & 15.364 & $0.01 *$ & \\
\hline
\end{tabular}

monitored targets. The upper limits for $M_{2} \sin i$ of hypothetical companions around the $R V$ constant BDs/VLMSs range between $0.1 M_{\text {Jup }}$ and $1.5 M_{\text {Jup }}$ (Table 3, upper part) assuming a circular orbit, a separation of 0.1 AU between companion and primary object, and adopting primary masses from Comerón et al. (1999, 2000). The adopted orbital separation of $0.1 \mathrm{AU}$ corresponds to orbital periods ranging between 30 and 70 days for them. As discussed in Guenther \& Wuchterl (2003), the snow-radius (i.e. the smallest orbital separation at which dust in a surrounding disk can condensate and giant planet formation by the core accretion model can occur) corresponds to orbital periods of 20-40 days for BDs/VLMSs as primaries. Thus, the $0.1 \mathrm{AU}$ separation adopted by us corresponds to about the snow-radius but is sometimes larger.

To conclude, nine BDs/VLMSs with spectral types M5-M8 and mass estimates $\lesssim 0.12 M_{\odot}$ show no $R V$ variability down to Jupiter mass planets for separations $\lesssim 0.1 \mathrm{AU}(0.25 \mathrm{AU}$ for B 34 and 1.2 $\mathrm{AU}$ for $\mathrm{Cha \textrm {H }} \alpha 4$ ). There is, of course, the possibility that existing companions have not been detected due to non-observations at the critical orbital phases. Furthermore, long-period companions may have been missed, since the sampled orbital periods for all of them but Cha $\mathrm{H} \alpha 4$ do not exceed 5 months.

\section{3. $R V$ variable objects}

For three objects, we found significant $R V$ variations, namely for the $\mathrm{BD}$ candidate $\mathrm{ChaH} \alpha 8$ (M6.5) and for the lowmass stars CHXR 74 (M4.5) and Sz 23 (M2.5), as shown in Fig. 9. The cross-correlation function for all of them is singlepeaked. The variability characteristic differs among the three objects. Sz 23 shows variability on time scales of days with no difference in the mean values of $R V \mathrm{~s}$ recorded in 2000 and in 2004. On the other hand, Cha H $\alpha 8$ and CHXR 74 show no or only smaller amplitude variations on time scales of days to weeks, whereas the mean $R V$ measured in 2000 differs significantly from the one measured years later, namely in 2002 for 

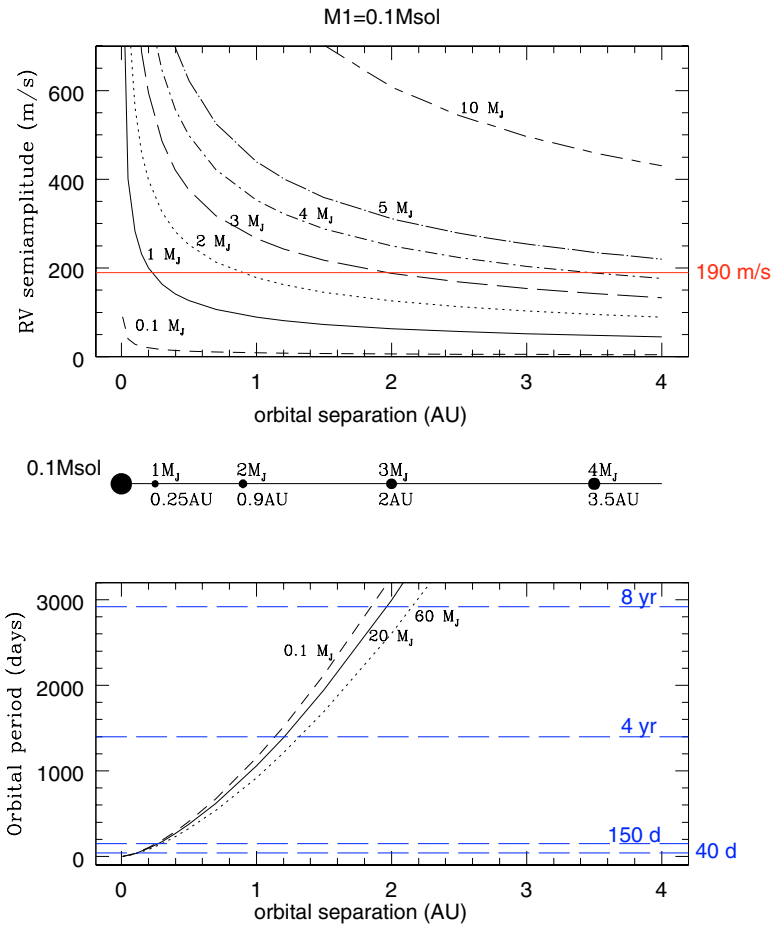

Fig. 1. Covered orbital separation ranges as function of $R V$ precision and time base of the obtained data for a $0.1 M_{\odot}$ primary. Top panel: $R V$ semiamplitude vs. orbital separation for different companion masses. With the average $R V$ precision of $190 \mathrm{~m} \mathrm{~s}^{-1}$ for primaries of $0.1 M_{\odot}$ achieved in this survey, a $1 M_{\text {Jup }}$ companion can be detected out to $0.25 \mathrm{AU}$, a $2 M_{\text {Jup }}$ companion out to $0.9 \mathrm{AU}$, a $3 M_{\text {Jup }}$ companion out to $2 \mathrm{AU}$, a $4 M_{\text {Jup }}$ companion out to $3.5 \mathrm{AU}$, and companions of $\geq 5 M_{\text {Jup }}$ at least out to $4 \mathrm{AU}$. However, due to a limited time base not all of the possible separation ranges have been covered yet. This is displayed in the bottom panel, which shows the orbital period vs separation exemplarily for companion masses of $0.1 M_{\mathrm{Jup}}$, $20 M_{\text {Jup }}$, and $60 M_{\text {Jup }}$. The orbital periods sampled in this survey so far vary among the different targets. For all targets, a period of 40 days has been covered, allowing the detection of substellar companions out to $0.1 \mathrm{AU}$. For some targets, periods of 150 days, $4 \mathrm{yr}$, and $8 \mathrm{yr}$ have also been probed and, thus, correspondingly larger separations, as indicated in the plot.

Cha $\mathrm{H} \alpha 8$ and in 2004 for CHXR 74, respectively, hinting at variability periods on the order of months or longer.

One possible explanation of the nature of these $R V$ variations is that they are caused by surface activity, since prominent surface spots can cause a shifting of the photo center at the rotation period. The upper limits for the rotational periods of Cha $\mathrm{H} \alpha$ 8, CHXR 74, and $\mathrm{Sz} 23$ are $1.9 \mathrm{~d}, 4.9 \mathrm{~d}$, and $2.1 \mathrm{~d}$, based on projected rotational velocities $v \sin i$ (Joergens \& Guenther 2001; cf. also Joergens et al. 2003). Thus, the timescale of the $R V$ variability of $\mathrm{Sz} 23$ is on the order of the rotation period and could be a rotation-induced phenomena, while the $R V$ variability of Cha $\mathrm{H} \alpha 8$ and $\mathrm{CHXR} 74$ on time scales of months to years cannot be explained as rotational modulation.

The other possibility is that the $R V$ variations are the Doppler shift caused by the gravitational force of orbiting companions. The poor sampling does not allow us to determine periods of the variations, but we can make some useful estimates. Based on the data for $\mathrm{Cha} \mathrm{H} \alpha 8\left(\sim 0.07 M_{\odot}\right)$, we suggest that its
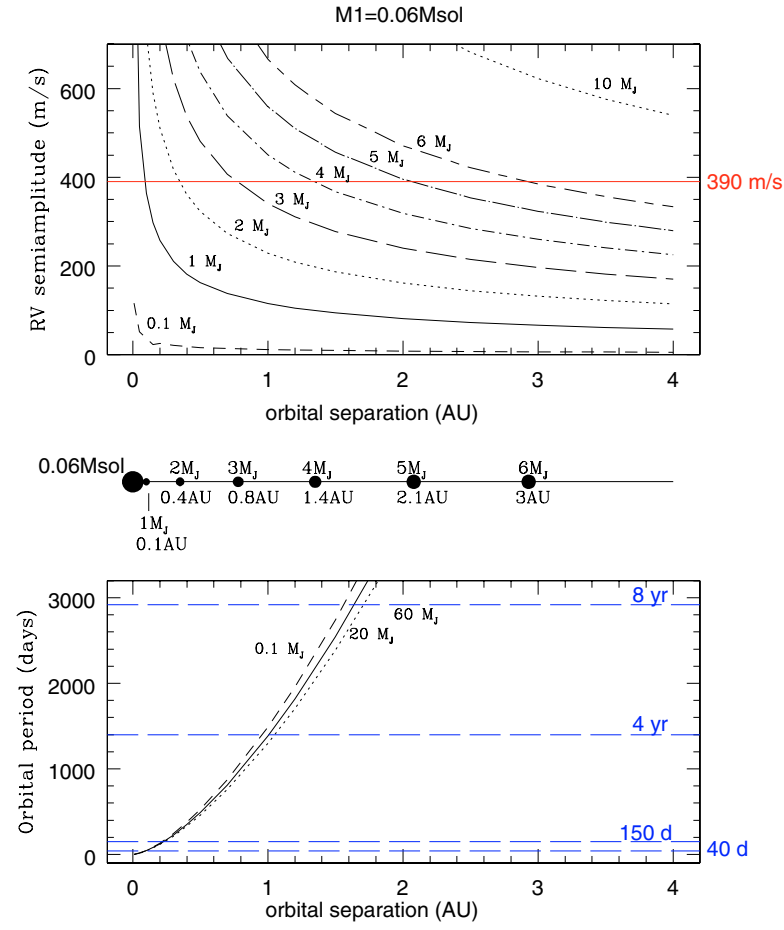

Fig. 2. Same as Fig. 1 but for a $0.06 M_{\odot}$ primary for which the $R V$ precision achieved in this survey is $390 \mathrm{~m} \mathrm{~s}^{-1}$ on average.

$R V$ period is at least 150 days, which transfers to an orbital separation of $z 0.2 \mathrm{AU}$, only weakly depending on the companion mass (cf. Fig. 2). The recorded half peak-to-peak $R V$ difference of $1.4 \mathrm{~km} \mathrm{~s}^{-1}$ is a lower limit for the $R V$ semiamplitude caused by a hypothetical companion. Thus, a companion causing these variations has to have a mass $M_{2} \sin i$ of at least $6 M_{\text {Jup }}$ when assuming a circular orbit. For CHXR 74, a period of $\gtrsim 200$ days would be consistent with the $R V$ data of 2000 and 2004 and would correspond to a separation of $\gtrsim 0.4 \mathrm{AU}$ and, thus, to a companion with mass $\gtrsim 15 M_{\text {Jup }}$.

\subsection{RV noise}

Figure 7 displays the measured difference in velocity $\Delta R V$ for all targets of the $R V$ survey in terms of the half size of the peakto-peak difference versus their mass, as adopted from Comerón et al. $(1999,2000)$. For the three $R V$ variable objects, $\Delta R V$ is above $1 \mathrm{~km} \mathrm{~s}^{-1}$ (top three data points in Fig. 7), and they are clearly separated from the $R V$ constant objects in this diagram. The apparent decrease in $\Delta R V$ with increasing mass observed for the $R V$ constant objects reflects the dependence of the $R V$ precision on the $\mathrm{S} / \mathrm{N}$ of the spectra. Interestingly, it also shows that this group of BDs/VLMSs with masses of $0.12 M_{\odot}$ and below display no significant $R V$ noise due to activity, which would cause systematic $R V$ errors with a $R V$ amplitude increasing with mass. Two of the variable objects ( $\mathrm{Cha} \mathrm{H} \alpha 8$ and CHXR 74) only show $R V$ variations above $1 \mathrm{~km} \mathrm{~s}^{-1}$ on longer time scales, while their short-term $R V$ differences are smaller. Therefore, we plot $\Delta R V$ recorded within one year (more precisely with time bases between 20 and 70 days) for each object in Fig. 8. For Cha $\mathrm{H} \alpha$ 1, 2, 3, 5, 6, 7, 12, B34, there is no 

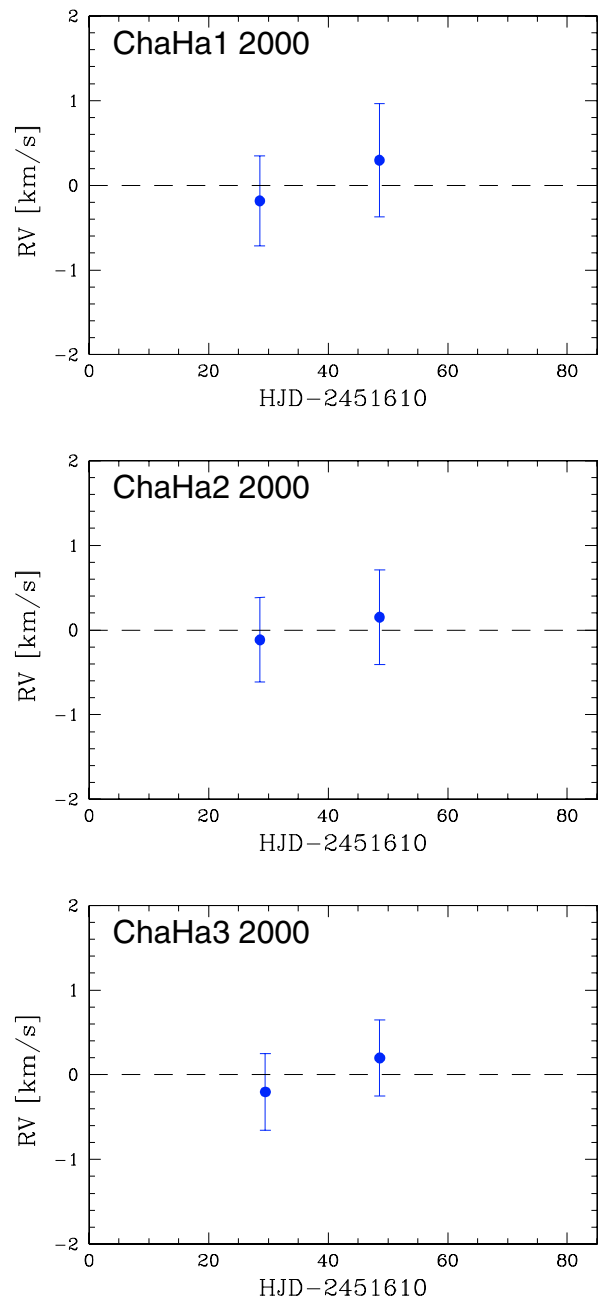

Fig. 3. $R V$ constant objects: relative $R V$ vs. time in Julian days for BDs/VLMSs in Cha I based on high-resolution UVES/VLT spectra. Error bars indicate $1 \sigma$ errors.

difference to Fig. 7, since data for these objects were generally recorded only within one year. For $\mathrm{Cha} \mathrm{H} \alpha 4$ and $\mathrm{Sz} 23$, there is also no difference because the recorded amplitudes of the shortterm and long-term variations do not differ. However, a difference occurs for Cha $\mathrm{H} \alpha 8$ and CHXR 74 because of the different $\Delta R V$ on the different time scales for these objects. Figure 8 shows that the relation of decreasing $R V$ difference with increasing mass in the BD regime continues to about $0.12 M_{\odot}$ and that it is reversed for higher masses. We therefore conclude that $\mathrm{BDs} / \mathrm{VLMSs}$ in Cha I display no significant $R V$ noise in the mass range below about $0.1 M_{\odot}$ and that roughly between 0.1 and $0.2 M_{\odot}$ the activity induced $R V$ noise is increasing drastically.

\subsection{Multiplicity}

The present $R V$ data hint at a very small multiplicity fraction of very young BDs/VLMSs for orbital periods roughly $\$ 40$ days and separations $\lessgtr 0.1 \mathrm{AU}$. All ten BDs/VLMSs $\left(\lessgtr 0.12 M_{\odot}\right)$ in this survey, are $R V$ constant with respect to companions in this parameter range in our observations. Among this subsample, there is only one (Cha $\mathrm{H} \alpha 8$ ) that shows signs of $R V$ variability,
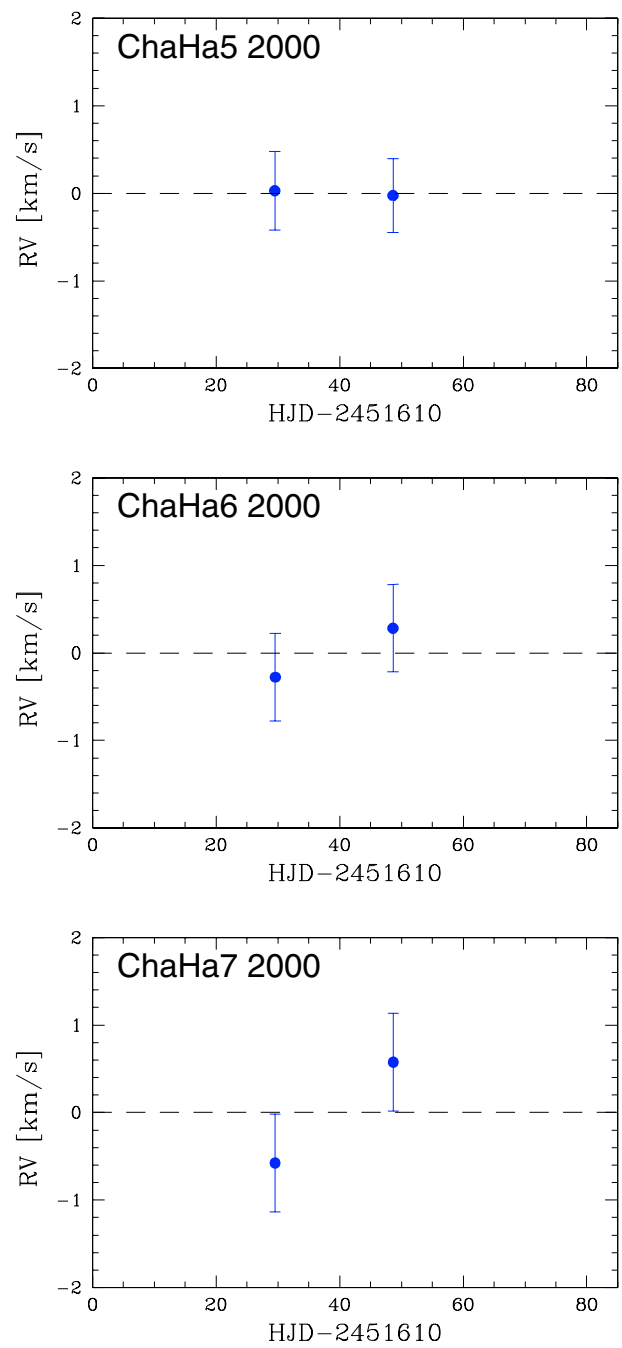

Fig. 4. $R V$ constant objects continued. See Fig. 3 .
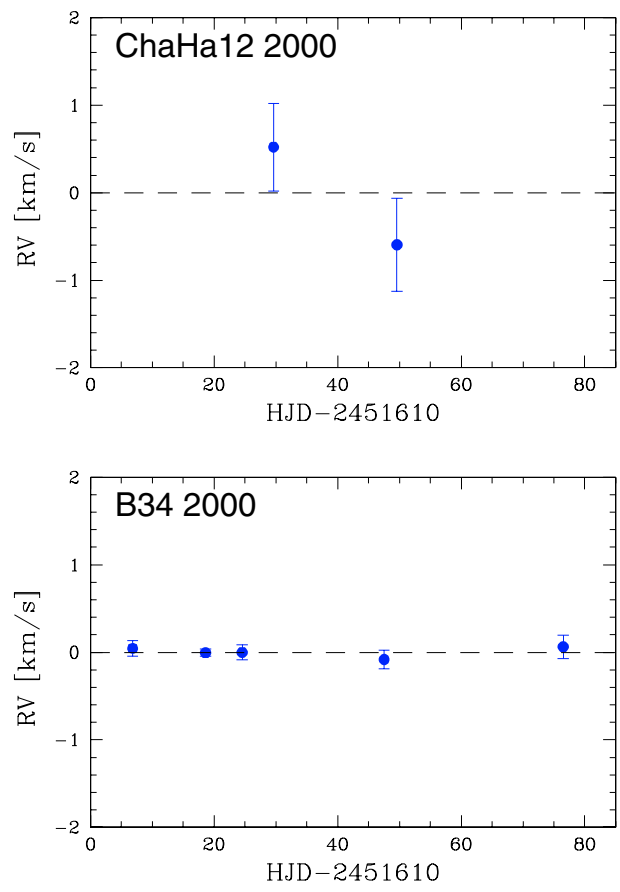

Fig. 5. $R V$ constant objects continued. See Fig. 3. 

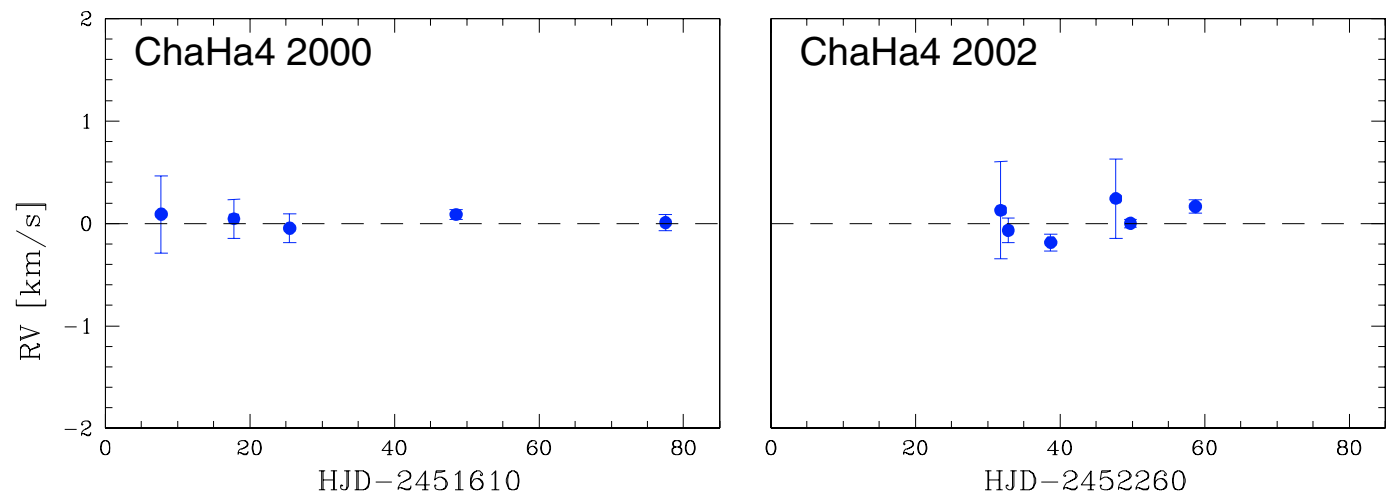

Fig. 6. $R V$ constant objects continued. See Fig. 3 .

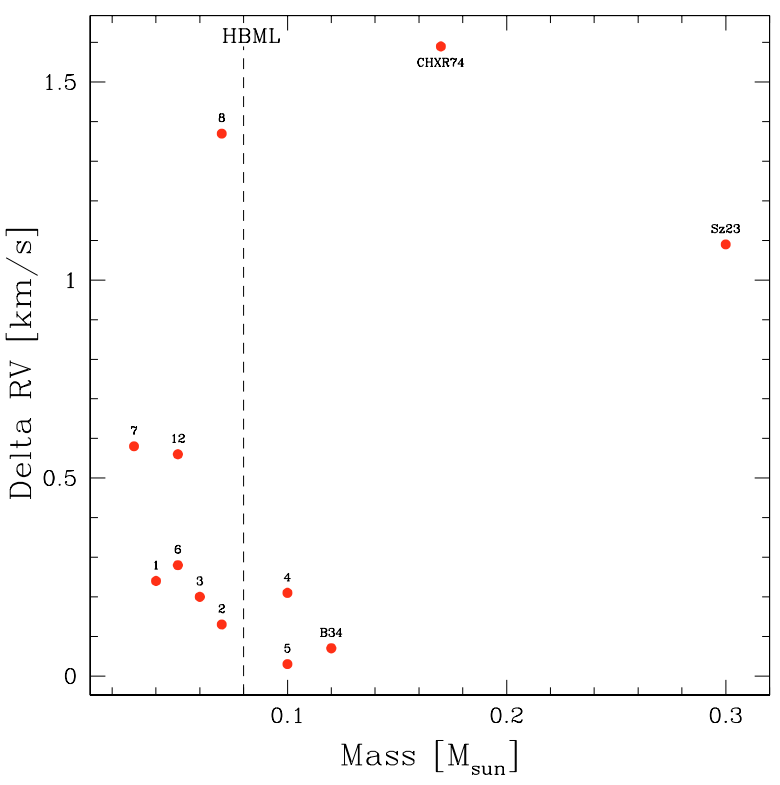

Fig. 7. $R V$ variations vs. object mass. Plotted are half peak-to-peak differences of the observed $R V \mathrm{~s}$. Each data point is labeled with the corresponding object name, the numbers denote the $\mathrm{Cha \textrm {H } \alpha}$ objects. The upper three data points represent the $R V$ variable objects with $\Delta R V$ above $1 \mathrm{~km} \mathrm{~s}^{-1}$. The remaining data points represent $R V$ constant objects. The decrease of $\Delta R V$ with increasing mass for the latter group indicates that they display no significant $R V$ noise due to activity, which would cause the opposite effect, i.e. an increasing $R V$ amplitude with mass. Mass estimates are from Comerón et al. (1999, 2000). HBML roughly indicates the theoretical "hydrogen burning mass limit".

namely on time scales of at least several months, corresponding to a separation of $0.2 \mathrm{AU}$ or larger. This separation range was probed so far for only two BDs/VLMSs, so no estimates of multiplicity rates in this separation range can be given yet.

The low-mass star CHXR $74\left(\sim 0.17 M_{\odot}\right)$ shows similar variability behavior as $\mathrm{Cha} \mathrm{H} \alpha 8$, i.e. small amplitude variations or no variations on time scales of days/weeks and larger amplitude $R V$ variability only on longer time scales of at least several months. From Figs. 7 and 8 , it can be seen that the recorded long-term $R V$ amplitudes of Cha $\mathrm{H} \alpha 8$ and CHXR 74 are significantly above the $R V$ noise level observed on short time scales. Furthermore, the timescales of the variability are

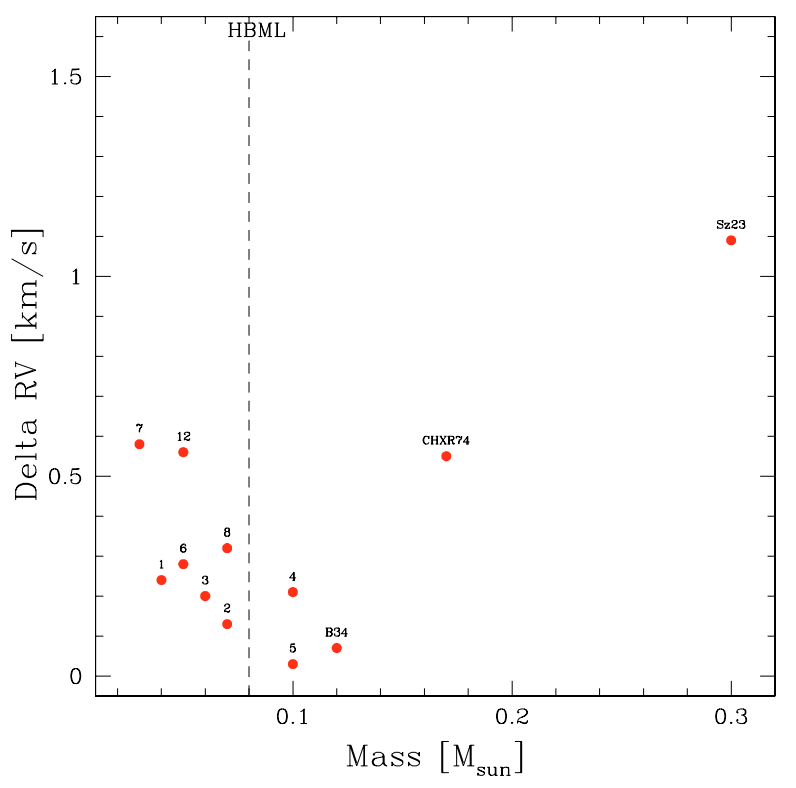

Fig. 8. $R V$ noise level: Same as Fig. 7 but only variations on time scales of days to weeks are considered. Differences to Fig. 7 occur for Cha $\mathrm{H} \alpha 8$ and $\mathrm{CHXR} 74$, whereas for all other objects, either data have been recorded anyway only within one year or the $R V$ variations observed on the short- and long-term do not differ in the recorded amplitude. It can be seen that the downward trend is reversed somewhere between 0.1 and $0.2 M_{\odot}$.

much too long to be caused by rotational modulation since the rotational periods are on the order of 2 days. The only other explanation could be a companion with a mass of several Jupiter masses or more, i.e. a supergiant planet or a brown dwarf. These observations hint that companions to young BDs and (very) low-mass stars might have periods of several months or longer, i.e. reside at orbital separations outside the snow-radius (cf. Guenther \& Wuchterl 2003).

The $R V$ survey probes the regions close to the central objects, in respect to the occurrence of companions, for all targets out to $0.1 \mathrm{AU}$ and for some even further out (2 AU maximum). At the moment, the limits in the separation range covered is set by the time base rather than by the $R V$ precision. Therefore, follow-up $R V$ measurements are planned. The small multiplicity fraction found for BDs/VLMSs in Cha I at small separations $(\lessgtr 0.1 \mathrm{AU})$ in this $R V$ survey is also supported by the results of 

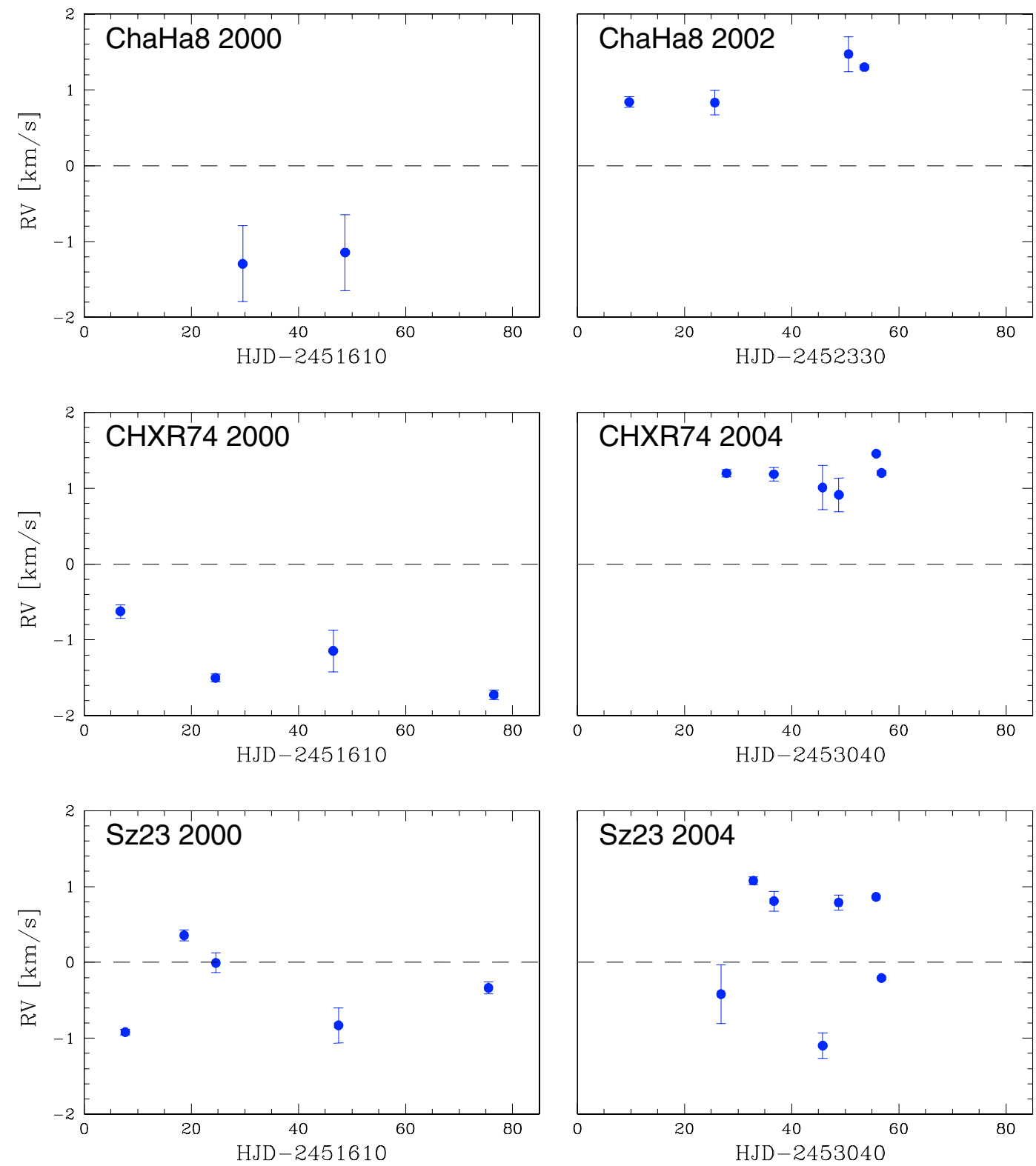

Fig. 9. $R V$ variable objects: relative $R V$ vs. time in Julian days for the $\mathrm{BD}$ candidate Cha $\mathrm{H} \alpha 8$ and the low-mass stars $\mathrm{CHXR} 74$ and $\mathrm{Sz} 23$ in Cha I based on UVES/VLT spectra. Error bars indicate $1 \sigma$ errors.

a direct imaging search for wide ${ }^{3}$ (planetary or brown dwarf) companions to mostly the same targets, namely Cha $\mathrm{H} \alpha 1-12$, by Neuhäuser et al. $(2002,2003)$, who find a multiplicity fraction of $\lesssim 10 \%$.

The absolute $R V \mathrm{~s}$ that are determined based on UVES spectra for Cha $\mathrm{H} \alpha 1,2,3,4,5,7, \mathrm{Sz} 23, \mathrm{~B} 34$, and CHXR 74 are consistent with moderately precise $R V \mathrm{~s}$ measured by Neuhäuser \& Comerón (1999) based on medium-resolution spectra within 1.2 times the errors. The $R V$ measured by these authors for $\mathrm{Cha} \mathrm{H} \alpha$ 8, for which we find significant $R V$ variability, is also discrepant by 1.6 times the errors with the $R V$ we derived for this object in 2004. Furthermore, the $R V$ they find for $\mathrm{Cha \textrm {H } \alpha} 6$ is discrepant with our value by 1.9 times the

\footnotetext{
3 The exact separation ranges covered depend on the mass and are e.g. $>50 \mathrm{AU}$ for a $20 M_{\text {Jup }}$ in orbit around a $60 M_{\text {Jup }}$, or $>300 \mathrm{AU}$ for a $1 M_{\text {Jup }}$ in orbit around a $60 M_{\text {Jup }}$.
}

errors, which might be a hint of a spectroscopic companion also around $\mathrm{Cha \textrm {H } \alpha} 6$.

\subsection{Brown dwarf desert}

It was one of the surprising results of the $R V$ surveys for extrasolar planets around solar-like stars that there is an almost complete absence of companions with BD masses in close orbits $(<3-5 \mathrm{AU})$, while at least $6.6 \%$ of the solar-like stars have planets $\left(<13 M_{\text {Jup }}\right)$ and about $15 \%$ of them have stellar companions (e.g. Halbwachs et al. 2000; Mazeh et al. 2003; Marcy et al. 2005b). Whatever physical reason causes the BD desert in the formation of solar-like stars, it should also be present in terms of mass ratio for primaries of significantly lower or higher mass, as long as they are formed by the same mechanism. For example, if the formation of BDs is a scaled-down 
Table 3. $R V$ differences and estimates of companion masses. $\Delta R V$ gives half peak-to-peak differences of the observed $R V \mathrm{~s}$ listed in Tables 1 and 2. For $R V$ constant objects in this survey (upper part of the table), $\Delta R V$ is an upper limit for the $R V$ semiamplitude of hypothetical companions missed due to $R V$ precision. For them, the last column lists upper limits for the companion minimum mass $M_{2} \sin i$ derived by assuming a semimajor axis of $0.1 \mathrm{AU}$ and circular orbits. For $R V$ variable objects (lower part of table), the recorded $R V$ differences are lower limits for the $R V$ amplitudes of hypothetical companions. For them, the last column gives a lower limit for the companion minimum mass $M_{2} \sin i$ for separations of $0.2 \mathrm{AU}(\mathrm{Cha} \mathrm{H} \alpha$ ) and 0.4 AU (CHXR 74, Sz 23), resp. Primary masses are taken from Comerón et al. (1999, 2000).

\begin{tabular}{lcc}
\hline \hline Object & $\begin{array}{c}\Delta R V \\
{\left[\mathrm{~km} \mathrm{~s}^{-1}\right]}\end{array}$ & $\begin{array}{c}M_{2} \sin i \\
{\left[M_{\text {Jup }}\right]}\end{array}$ \\
\hline$R V$ constant: & & $\max . M_{2} \sin i$ \\
\hline Cha H $\alpha 1$ & 0.24 & 0.6 \\
Cha H $\alpha 2$ & 0.13 & 0.4 \\
Cha H $\alpha 3$ & 0.20 & 0.6 \\
Cha H $\alpha 4$ & 0.21 & 0.8 \\
Cha H $\alpha 5$ & 0.03 & 0.1 \\
Cha H $\alpha 6$ & 0.28 & 0.6 \\
Cha H $\alpha 7$ & 0.58 & 1.1 \\
Cha H $\alpha 12$ & 0.56 & 1.5 \\
B 34 & 0.07 & 0.3 \\
\hline$R V$ variable: & & min. $M_{2} \sin i$ \\
\hline Cha H $\alpha$ 8 & 1.37 & 6 \\
CHXR 74 & 1.59 & 15 \\
Sz 23 & 1.09 & 13 \\
\hline
\end{tabular}

low-mass star formation process (i.e. cloud fragmentation and direct collapse of small cloud cores above the opacity limit), the BD desert should be found in a scaled-down version shifted to lower companion masses also around them.

In order to quantify this, we looked at the distribution of mass ratios for known $R V$ planets around solar-like stars and inferred from it that the lower boundary of the BD desert is at $M_{2} / M_{1} \approx 0.02$. Its upper boundary, on the other hand, is not as well-defined. The lowest mass ratio known for a stellar spectroscopic companion to a solar-like star is 0.2 (Prato et al. 2002; Mazeh et al. 2003); however, as pointed out by Mazeh et al. (2003), stellar systems with $M_{2} / M_{1}<0.2-0.3$ have not been studied well yet. For the following consideration, a mass ratio of 0.08 is somewhat arbitrarily assumed as the upper value for the BD desert, but this is not confirmed by observations.

The BD desert around solar-like stars is now scaled down to the primary masses of the target BDs and VLMSs of this work $\left(0.03 \lessgtr M_{1} \lesssim 0.12 M_{\odot}\right)$. For the lowest mass primary studied here $\left(0.03 M_{\odot}\right)$, the BD desert would be shifted towards companion masses of $0.6-2.5 M_{\mathrm{Jup}}$, and for a $0.12 M_{\odot}$ primary towards $2.5-10 M_{\text {Jup }}$. Thus, the scaled-down equivalent to the $\mathrm{BD}$ desert around solar-like stars would be a giant planet desert around BD and VLMS primaries.
Our $R V$ survey started to test its presence around the targets in Cha I. For the orbital separations covered so far (for all targets $\lesssim 0.1 \mathrm{AU}$ and for some even larger with a maximum of $\lesssim 1.2 \mathrm{AU}$ for $M_{1} \lesssim 0.12 M_{\odot}$ ), companions in the "giant planet desert" are clearly detectable for primaries above $0.06 M_{\odot}$, whereas for the lowest mass primaries studied $\left(0.03-0.05 M_{\odot}\right)$ the sensitivity allows only a part of the "giant planet desert" around them to be probed. No hints of companions within these parameter ranges were found in the data. The lower mass limits roughly estimated for hypothetical companions around the $R V$ variable objects Cha $\mathrm{H} \alpha 8$ and $\mathrm{CHXR} 74$ would locate them just outside the "giant planet desert" around them, which is 1.5-5.9 $M_{\text {Jup }}$ for Cha $\mathrm{H} \alpha 8$ and 4-14 $M_{\text {Jup }}$ for CHXR 74, respectively; however, the assumed upper boundary is somewhat uncertain, as pointed out above.

So far, only a fraction of the orbital separations, for which the BD desert is established around solar-like stars $(<3-5 \mathrm{AU})$, has been probed yet. Larger separations will be explored by follow-up UVES observations.

For higher than solar-mass primaries, $R V$ surveys of $\mathrm{K}$ giants detected a much higher rate of close BD companions compared to solar-like stars (Frink et al. 2002; Hatzes et al. 2005; Setiawan 2005; Mitchell et al. 2005), which with only one exception all correspond to mass ratios $<0.02$, i.e. do not lie in the brown dwarf desert when scaled up for the higher primary masses.

\section{Conclusions and summary}

We have presented time-resolved high-resolution spectroscopic observations with UVES at the VLT of a population of very young BDs and (very) low-mass stars in the Cha I star-forming region. As they have an age of only a few million years, exploration allows insight into the formation and early evolution of BDs and of stars close to the substellar borderline. The $R V$ precision achieved in this $R V$ survey is sufficient for detecting companions down to Jupiter mass planets. The orbital periods sampled so far correspond to orbital separations of $\lesssim 0.1 \mathrm{AU}$ (for some also to larger separations up to almost $3 \mathrm{AU})$. Therefore, it allows us to probe planet formation at very young ages $(1-10 \mathrm{Myr})$, around very low-mass, partly substellar, primaries and at close orbital separations. This combination of primary mass range, primary spectral type, age, and separations has not been covered by previous surveys, which were either done by direct imaging, and, therefore, were only sensitive to larger separations or the primaries were of considerably larger mass or age.

The analysis of the UVES spectra reveals very constant $R V \mathrm{~s}$ on time scales of weeks to months for the majority of the targets, as well as $R V$ variability for three sources. The $R V$ constant objects are six BDs and three VLMSs $\left(M \lesssim 0.12 M_{\odot}\right.$, spectral types M5-M8), for which we estimate upper limits for masses of hypothetical companions in the range of $0.1 M_{\mathrm{Jup}}$ to $1.5 M_{\text {Jup }}$ by assuming orbital separations of $0.1 \mathrm{AU}$, which corresponds to the sampled periods (40 days). The data show that this group displays no significant $R V$ noise due to activity down to the precision necessary to detect Jupiter mass planets. This demonstrates that BDs/VLMSs in Cha I are suitable 
targets when using the $R V$ technique to search for planets. This opens up a new parameter range for the search for extrasolar planets, namely very young planets at very close separations. Even with the unprecedented angular resolution of the new generation of optical and near-IR ground-based interferometers, like the VLT Interferometer, the separation ranges for which the $R V$ method is sensitive are not covered by existing and near-future instruments for planetary mass companions in nearby star forming regions.

Three objects exhibit significant $R V$ variations with peakto-peak $R V$ differences of $2-3 \mathrm{~km} \mathrm{~s}^{-1}$, namely the BD candidate $\mathrm{Cha} \mathrm{H} \alpha 8$ (M6.5) and the low-mass stars CHXR 74 (M4.5, $\left.\sim 0.17 M_{\odot}\right)$ and $\mathrm{Sz} 23\left(\mathrm{M} 2.5, \sim 0.3 M_{\odot}\right)$. A possible explanation for the short-term $R V$ variations on time scales of days for $\mathrm{Sz} 23$, which is the highest mass object in the sample, are surface spots. The other two variable objects, Cha $\mathrm{H} \alpha 8$ and CHXR 74, show different variability behavior. They display only very small or no $R V$ variability on time scales of days to weeks but significant $R V$ variations on time scales of months or longer, which cannot be explained by rotational modulation and, therefore, it hints at orbiting companions. The poor phase coverage does not allow determination of orbital parameters for the hypothetical companions. However, the $R V$ data for Cha $\mathrm{H} \alpha 8$ suggest that its period is at least five months, which would correspond to orbital separations of at least $0.2 \mathrm{AU}$. Based on these numbers, the detected $R V$ variations of Cha $\mathrm{H} \alpha 8$ could be caused by a $6 M_{\text {Jup }}$ or a more massive companion. For CHXR 74, the data suggest a period of 7 months or longer ( $\gtrsim 0.4 \mathrm{AU})$ and a companion of at least $15 M_{\text {Jup }}$. In order to explore the nature of the detected $R V$ variations, follow-up $R V$ measurements of CHXR 74 and $\mathrm{Cha \textrm {H }} \alpha 8$ will be performed. If confirmed as planetary systems, they would be exceptional, because they would contain the lowest mass primaries and the first BD with an $R V$ planet. With an age of a few million years, they would also harbor by far the youngest extrasolar $R V$ planet found to date. This would provide empirical constraints for planet and $\mathrm{BD}$ formation and early evolution.

The $R V$ data presented here indicate that the multiplicity fraction of very young BDs and (very) low-mass stars is very small for orbital separations below $0.1 \mathrm{AU}$, which corresponds to about the snow line around the targets (Guenther \& Wuchterl 2003). The subsample of ten BDs/VLMSs with masses $\lesssim 0.12 M_{\odot}$, are $R V$-constant for orbital periods below 40 days. In addition, this is true for $\mathrm{Cha} \mathrm{H} \alpha 4$ and B 34 for periods below 150 days and for $\mathrm{Cha} \mathrm{H} \alpha 4$ for periods below 4 years. Only one object of this group, namely Cha $\mathrm{H} \alpha$, turned out to be variable on time scales of at least 150 days. This object and the higher mass CHXR 74 (not included in the above considered subsample) hint at the possibility that companions to young BDs/VLMSs have periods of at least several months. Such a time scale was not covered for a substantial part of the targets. Therefore a multiplicity rate cannot be determined yet. Follow-up $R V$ measurements will probe these time scales for the remaining targets.

Furthermore, we show that the scaled-down equivalent to the BD desert found around solar-like stars would be a "giant planet desert" around BDs/VLMSs if formed by the same mechanism. For example, for a $0.03 M_{\odot}$ primary, the deserted companion mass region would be $0.6-2.5 M_{\text {Jup }}$ and, for a $0.12 M_{\odot}$ primary, $2.5-10 M_{\mathrm{Jup}}$. The present $R V$ data test the existence of such a 'giant planet desert' for the targets. For the orbital separations covered so far, companions in the "giant planet desert" are clearly detectable for primaries above $0.06 M_{\odot}$, whereas for the lowest mass primaries studied $\left(0.03-0.05 M_{\odot}\right)$, the sensitivity allows probing only a part of it. So far, no hints have been found of companions in these mass ranges.

At much larger separations, a direct imaging search for wide (planetary or brown dwarf) companions to mostly the same targets also found a very small multiplicity fraction (Neuhäuser et al. 2002, 2003). There still remains a significant gap in the separation ranges studied, which will be probed partly by the planned follow-up $R V$ measurements and is partly only accessible with high-resolving AO imaging (NACO/VLT), or it requires interferometric techniques (e.g. AMBER at the VLTI).

Acknowledgements. I am grateful to Ralph Neuhäuser and Eike Guenther for assistance in the early stages of this project. I would also like to thank the referee, Fernando Comerón, for very helpful comments that improved the paper significantly. This work made use of software by Eike Guenther to calculate spectroscopic orbits. I am pleased to acknowledge the excellent work of the ESO staff at Paranal, who took all the UVES observations the present work is based on in service mode. I thank Francesca Primas, Ferdinando Patat, Benoit Pirenne, and Eric Louppe from ESO Garching for kind and efficient help in various stages of the observation preparation and data handling. Furthermore, I acknowledge a grant by the Deutsche Forschungsgemeinschaft (Schwerpunktprogramm "Physics of star formation") during the beginning of the project, as well as current financial support by a Marie Curie Fellowship of the European Community programme "Structuring the European Research Area" under contract number FP6-501875. Part of the earlier work was carried out at the Max-Planck-Institute for Extraterrestrial Physics, Garching, Germany.

\section{References}

Balthasar, H., Thiele, U., \& Wöhl, H. 1982, A\&A, 114, 357

Basri, G., \& Martín, E. L. 1999, ApJ, 118, 2460

Bouy, H., Brandner, W., Martín, E. L., et al. 2003, AJ, 126, 1526

Bouy, H., Brandner, W., Martín, E. L., et al. 2004, A\&A, 424, 213

Butler, P., Vogt, S., Marcy, G., et al. 2004, ApJ, 617, 580

Caccin, B., Cavallini, F., Ceppatelli, G., Righini, A., \& Sambuco, A. M. 1985, A\&A, 149, 357

Chauvin, G., Lagrange, A.-M., Dumas, C., et al. 2004, A\&A, 425, L29

Chauvin, G., Lagrange, A.-M., Dumas, C., et al. 2005, A\&A, 438, L25

Comerón, F., Rieke, G. H., \& Neuhäuser, R. 1999, A\&A, 343, 477

Comerón, F., Neuhäuser, R., \& Kaas, A. A. 2000, A\&A, 359, 269

Dekker, H., D’Odorico, S., Kaufer, A., Delabre, B., \& Kotzlowski, H. 2000, in SPIE, ed. M. Iye, \& A. Moorwood, 4008, 534

Delfosse, X., Forveille, T., Perrier, C., \& Mayor, M. 1998, A\&A, 331, 581

Frink, S., Mitchell, D. S., Quirrenbach, A., et al. 2002, ApJ, 576, 478

Guenther, E. W., \& Wuchterl, G. 2003, A\&A, 401, 677

Halbwachs, J. L., Arenou, F., Mayor, M., Udry, S., \& Queloz, D. 2000, A\&A, 355, 581

Hatzes, A. P., Guenther, E. W., Endl, M., et al. 2005, A\&A, 437, 743 
Joergens, V., \& Guenther, E. 2001, A\&A, 379, L9

Joergens, V. 2003, Ph.D. Thesis, Ludwigs-Maximilians Universität München

Joergens, V., Fernández, M., Carpenter, J. M., \& Neuhäuser, R. 2003, ApJ, 594, 971

Joergens, V. 2005, Rev. Mod. Astron., ed. S. Roeser (Weinheim: Wiley), 18, 216

Joergens, V. 2006, A\&A, in press [arXiv: astro-ph/0509462]

Kürster, M., Endl, M., Els, S., et al. 2000, A\&A, 353, L33

Luhman, K. L. 2004, ApJ, 614, 398

Marcy, G., Butler, P., Vogt, S., Fischer, D., \& Lissauer, J. 1998, ApJ, 505, L147

Marcy, G., Butler, P., Fischer, D., et al. 2001, ApJ, 556, 296

Marcy, G., Butler, R. P., Fischer, D., et al. 2005b, Progress of Theoretical Physics Supplement, No. 158 [arXiv: astro-ph/0505003]
Marcy, G., Butler, P., Vogt, S., et al. 2005a, ApJ, 619, 570

Mazeh, T., Simon, M., Prato, L., Markus, B., \& Zucker, S. 2003, ApJ, 599,1344

Mitchell, D. S., Frink, S., Quirrenbach, A., et al. 2005, submitted

Moutou, C., Mayor, M., Bouchy, F., et al. 2005, A\&A, 439, 367

Neuhäuser, R., \& Comerón, F. 1998, Science, 282, 83

Neuhäuser, R., \& Comerón, F. 1999, A\&A, 350, 612

Neuhäuser, R., Brandner, W., Alves, J., Joergens, V., \& Comerón, F. 2002, A\&A, 384, 999

Neuhäuser, R., Brandner, W., \& Guenther, E. 2003, IAUS, 211, 309, ed. E. Martín

Neuhäuser, R., Guenther, E. W., Wuchterl, G., et al. 2005, A\&A, 435, L13

Prato, L., Simon, M., Mazeh, T., et al. 2002, ApJ, 569, 863

Santos, N. C., Bouchy, F., Mayor, M., et al. 2004, A\&A, 426, L19

Setiawan, J., Rodmann, J., da Silva, L., et al. 2005, A\&A, 437, L31 


\section{Online Material}


V. Joergens: Radial velocity survey of brown dwarfs and very low-mass stars in Cha I with UVES at the VLT, Online Material $p 2$

\section{Appendix A: Observing logs UVES spectra}

Table A.1. Observing log: UVES spectroscopy of BDs and (very) low-mass stars in Cha I. Given are the date of the observation, heliocentric Julian day (HJD) at the middle of the exposure, the exposure time, and the radial velocity $R V$ for each spectrum.

\begin{tabular}{|c|c|c|c|c|}
\hline Object & Date & HJD & $\begin{array}{c}\text { Exposure } \\
{[\mathrm{s}]}\end{array}$ & $\begin{array}{c}R V \\
{\left[\mathrm{~km} \mathrm{~s}^{-1}\right]}\end{array}$ \\
\hline \multirow[t]{2}{*}{ Cha $\mathrm{H} \alpha 1$} & 2000 Apr. 04 & 2451638.56395 & $2 \times 1650$ & 16.167 \\
\hline & 2000 Apr. 24 & 2451658.57346 & $2 \times 1650$ & 16.648 \\
\hline \multirow[t]{2}{*}{ Cha $\mathrm{H} \alpha 2$} & 2000 Apr. 04 & 2451638.59431 & $1 \times 1029$ & 16.015 \\
\hline & 2000 Apr. 24 & 2451658.60407 & $1 \times 1029$ & 16.282 \\
\hline \multirow[t]{2}{*}{ Cha $\mathrm{H} \alpha 3$} & 2000 Apr. 04 & 2451639.49340 & $1 \times 899$ & 14.357 \\
\hline & 2000 Apr. 24 & 2451658.61991 & $1 \times 899$ & 14.758 \\
\hline \multirow[t]{24}{*}{$\mathrm{ChaH} \alpha 4$} & 2000 Mar. 14 & 2451617.72337 & $1 \times 2200$ & 15.176 \\
\hline & 2000 Mar. 14 & 2451617.74954 & $1 \times 2200$ & 14.642 \\
\hline & 2000 Mar. 24 & 2451627.79079 & $1 \times 2200$ & 14.732 \\
\hline & 2000 Mar. 24 & 2451627.81697 & $1 \times 2200$ & 14.999 \\
\hline & 2000 Mar. 31 & 2451635.51085 & $1 \times 2200$ & 14.773 \\
\hline & 2000 Apr. 23 & 2451658.50840 & $1 \times 2200$ & 14.941 \\
\hline & 2000 Apr. 23 & 2451658.53460 & $1 \times 2200$ & 14.875 \\
\hline & 2000 May 22 & 2451687.49289 & $1 \times 2200$ & 14.774 \\
\hline & 2000 May 23 & 2451687.51900 & $1 \times 2200$ & 14.885 \\
\hline & 2002 Jan. 17 & 2452291.73197 & $2 \times 1100$ & 14.761 \\
\hline & 2002 Jan. 17 & 2452291.75976 & $2 \times 1100$ & 15.614 \\
\hline & 2002 Jan. 17 & 2452291.79010 & $2 \times 1100$ & 14.661 \\
\hline & 2002 Jan. 17 & 2452291.81794 & $2 \times 1100$ & 15.260 \\
\hline & 2002 Jan. 17 & 2452291.84586 & $2 \times 1100$ & 14.450 \\
\hline & 2002 Jan. 18 & 2452292.81113 & $2 \times 1100$ & 14.669 \\
\hline & 2002 Jan. 18 & 2452292.83902 & $2 \times 1100$ & 14.839 \\
\hline & 2002 Jan. 24 & 2452298.69149 & $2 \times 1100$ & 14.575 \\
\hline & 2002 Jan. 24 & 2452298.71931 & $2 \times 1100$ & 14.694 \\
\hline & 2002 Feb. 02 & 2452307.70591 & $2 \times 1100$ & 15.338 \\
\hline & 2002 Feb. 02 & 2452307.73367 & $2 \times 1100$ & 14.790 \\
\hline & 2002 Feb. 04 & 2452309.72831 & $2 \times 1100$ & 14.821 \\
\hline & 2002 Feb. 04 & 2452309.75613 & $2 \times 1100$ & 14.820 \\
\hline & 2002 Feb. 13 & 2452318.70523 & $2 \times 1100$ & 14.939 \\
\hline & 2002 Feb. 13 & 2452318.73306 & $2 \times 1100$ & 15.031 \\
\hline \multirow[t]{2}{*}{ Cha $\mathrm{H} \alpha 5$} & 2000 Apr. 05 & 2451639.51485 & $1 \times 800$ & 15.499 \\
\hline & 2000 Apr. 24 & 2451658.63522 & $1 \times 800$ & 15.446 \\
\hline \multirow[t]{2}{*}{ Cha H $\alpha 6$} & 2000 Apr. 05 & 2451639.58967 & $1 \times 1029$ & 16.093 \\
\hline & 2000 Apr. 24 & 2451658.65099 & $1 \times 1029$ & 16.652 \\
\hline \multirow[t]{2}{*}{ Cha $\mathrm{H} \alpha 7$} & 2000 Apr. 05 & 2451639.55225 & $2 \times 2150$ & 16.513 \\
\hline & 2000 Apr. 24 & 2451658.68756 & $2 \times 2150$ & 17.664 \\
\hline \multirow[t]{10}{*}{ Cha $\mathrm{H} \alpha 8$} & 2000 Apr. 05 & 2451639.61095 & $1 \times 1599$ & 14.787 \\
\hline & 2000 Apr. 24 & 2451658.72597 & $1 \times 1600$ & 14.935 \\
\hline & 2002 Mar. 06 & 2452339.67846 & $1 \times 1785$ & 16.972 \\
\hline & 2002 Mar. 06 & 2452339.70084 & $1 \times 1785$ & 16.868 \\
\hline & 2002 Mar. 22 & 2452355.64149 & $1 \times 1785$ & 16.795 \\
\hline & 2002 Mar. 22 & 2452355.66378 & $1 \times 1785$ & 17.028 \\
\hline & 2002 Apr. 16 & 2452380.60528 & $1 \times 1785$ & 17.391 \\
\hline & 2002 Apr. 16 & 2452380.62764 & $1 \times 1785$ & 17.710 \\
\hline & 2002 Apr. 19 & 2452383.56440 & $1 \times 1785$ & 17.356 \\
\hline & 2002 Apr. 19 & 2452383.58690 & $1 \times 1785$ & 17.402 \\
\hline
\end{tabular}

Table A.2. Observing log: UVES spectroscopy continued. See Table A.1 for details.

\begin{tabular}{|c|c|c|c|c|}
\hline Object & Date & HJD & $\begin{array}{c}\text { Exposure } \\
{[\mathrm{s}]}\end{array}$ & $\begin{array}{c}R V \\
{\left[\mathrm{~km} \mathrm{~s}^{-1}\right]}\end{array}$ \\
\hline \multirow[t]{2}{*}{ Cha $\mathrm{H} \alpha 12$} & 2000 Apr. 05 & 2451639.63487 & $1 \times 1599$ & 15.021 \\
\hline & 2000 Apr. 25 & 2451659.59469 & $1 \times 1600$ & 13.905 \\
\hline \multirow[t]{9}{*}{ B 34} & 2000 Mar. 13 & 2451616.82387 & $1 \times 1350$ & 15.730 \\
\hline & 2000 Mar. 13 & 2451616.84023 & $1 \times 1350$ & 15.859 \\
\hline & 2000 Mar. 25 & 2451628.61377 & $1 \times 2700$ & 15.746 \\
\hline & 2000 Mar. 31 & 2451634.54665 & $1 \times 1350$ & 15.687 \\
\hline & 2000 Mar. 31 & 2451634.56298 & $1 \times 1350$ & 15.810 \\
\hline & 2000 Apr. 23 & 2451657.52652 & $1 \times 1350$ & 15.591 \\
\hline & 2000 Apr. 23 & 2451657.54287 & $1 \times 1350$ & 15.743 \\
\hline & 2000 May 21 & 2451686.50566 & $1 \times 1350$ & 15.720 \\
\hline & 2000 May 22 & 2451686.52202 & $1 \times 1350$ & 15.908 \\
\hline \multirow[t]{20}{*}{$\mathrm{CH} \times \mathrm{R} 74$} & 2000 Mar. 13 & 2451616.78332 & $1 \times 600$ & 15.313 \\
\hline & 2000 Mar. 13 & 2451616.79097 & $1 \times 600$ & 15.439 \\
\hline & 2000 Mar. 31 & 2451634.51710 & $1 \times 600$ & 14.537 \\
\hline & 2000 Mar. 31 & 2451634.52473 & $1 \times 600$ & 14.460 \\
\hline & 2000 Apr. 22 & 2451656.50861 & $1 \times 600$ & 15.048 \\
\hline & 2000 Apr. 22 & 2451656.51633 & $1 \times 600$ & 14.660 \\
\hline & 2000 May 21 & 2451686.47878 & $1 \times 600$ & 14.320 \\
\hline & 2000 May 21 & 2451686.48644 & $1 \times 600$ & 14.232 \\
\hline & 2004 Mar. 03 & 2453067.82538 & $1 \times 630$ & 17.234 \\
\hline & 2004 Mar. 03 & 2453067.83374 & $1 \times 630$ & 17.157 \\
\hline & 2004 Mar. 12 & 2453076.66087 & $1 \times 630$ & 17.123 \\
\hline & 2004 Mar. 12 & 2453076.66888 & $1 \times 630$ & 17.244 \\
\hline & 2004 Mar. 21 & 2453085.76620 & $1 \times 630$ & 16.805 \\
\hline & 2004 Mar. 21 & 2453085.77424 & $1 \times 630$ & 17.213 \\
\hline & 2004 Mar. 24 & 2453088.79419 & $1 \times 630$ & 16.756 \\
\hline & 2004 Mar. 24 & 2453088.80224 & $1 \times 630$ & 17.068 \\
\hline & 2004 Mar. 31 & 2453095.77639 & $1 \times 630$ & 17.447 \\
\hline & 2004 Mar. 31 & 2453095.78445 & $1 \times 630$ & 17.460 \\
\hline & 2004 Mar. 01 & 2453096.77199 & $1 \times 630$ & 17.218 \\
\hline & 2004 Mar. 01 & 2453096.78001 & $1 \times 630$ & 17.181 \\
\hline \multirow[t]{22}{*}{ Sz 23} & 2000 Mar. 14 & 2451617.68093 & $2 \times 1350$ & 14.652 \\
\hline & 2000 Mar. 25 & 2451628.66914 & $2 \times 1350$ & 15.926 \\
\hline & 2000 Mar. 31 & 2451634.58325 & $1 \times 1350$ & 15.656 \\
\hline & 2000 Mar. 31 & 2451634.59958 & $1 \times 1350$ & 15.472 \\
\hline & 2000 Apr. 22 & 2451657.48819 & $1 \times 1350$ & 14.575 \\
\hline & 2000 Apr. 22 & 2451657.50452 & $1 \times 1350$ & 14.905 \\
\hline & 2000 May 20 & 2451685.47992 & $1 \times 1350$ & 15.288 \\
\hline & 2000 May 20 & 2451685.49632 & $1 \times 1350$ & 15.178 \\
\hline & 2004 Mar. 02 & 2453066.79564 & $1 \times 1300$ & 15.431 \\
\hline & 2004 Mar. 02 & 2453066.81152 & $1 \times 1300$ & 14.873 \\
\hline & 2004 Mar. 08 & 2453072.83264 & $1 \times 1300$ & 16.610 \\
\hline & 2004 Mar. 08 & 2453072.84841 & $1 \times 1300$ & 16.684 \\
\hline & 2004 Mar. 12 & 2453076.68615 & $1 \times 1300$ & 16.283 \\
\hline & 2004 Mar. 12 & 2453076.70222 & $1 \times 1300$ & 16.470 \\
\hline & 2004 Mar. 21 & 2453085.79265 & $1 \times 1300$ & 14.591 \\
\hline & 2004 Mar. 21 & 2453085.80853 & $1 \times 1300$ & 14.353 \\
\hline & 2004 Mar. 24 & 2453088.76236 & $1 \times 1300$ & 16.430 \\
\hline & 2004 Mar. 24 & 2453088.77820 & $1 \times 1300$ & 16.289 \\
\hline & 2004 Mar. 31 & 2453095.74401 & $1 \times 1300$ & 16.447 \\
\hline & 2004 Mar. 31 & 2453095.76013 & $1 \times 1300$ & 16.416 \\
\hline & 2004 Apr. 01 & 2453096.74102 & $1 \times 1300$ & 15.359 \\
\hline & 2004 Apr. 01 & 2453096.75691 & $1 \times 1300$ & 15.369 \\
\hline
\end{tabular}

